ORIGINAL RESEARCH

\title{
Risk Determinants of Acute Mountain Sickness and Summit Success on a 6-Day Ascent of Mount Kilimanjaro $(5895 \mathrm{~m})$
}

\author{
James S. Lawrence, BMedSci, BMBS; Stephen A. Reid, PhD \\ From the North Sydney Sports Medicine Centre, St. Leonards, Australia (Dr Lawrence); and the Sports Medicine Practice, Hobart, Australia (Dr Reid).
}

\begin{abstract}
Objective.-The aims of this study were to assess incidence of acute mountain sickness (AMS) and summit success on a 6-day ascent profile of Mt. Kilimanjaro and evaluate potential risk factors for these outcomes.

Methods.-All trekkers through a single Australian tour company between August 2012 and July 2014 were included. Participants ascended via the Rongai route and attempted the summit on day 6. Daily assessments were made using the self-reported Lake Louise score (LLS) questionnaire. Two different AMS diagnostic criteria (LLS $\geq 3$ and LLS $\geq 5$ ) were used for data analysis. Risk factors for development of AMS and summit success were analyzed.

Results. - Over the 24-month period a total of 175 participants undertook the trek. Incidence of AMS was $52.6 \%$ (LLS $\geq 3$ ) and $22.9 \%$ (LLS $\geq 5$ ). Summit success was $88 \%$. Age, sex, body mass index, and acetazolamide use were not associated with risk of AMS development. Age $\geq 40$ years $(P=.0002)$ and female sex $(P=.0004)$ were both significantly associated with reduced summit success rate.

Conclusions.- - Our cohort found a lower incidence of AMS and better summit success on a 6-day ascent of Mt Kilimanjaro than previously described in other groups on 4- and 5-day ascents. Female sex and age $\geq 40$ years both predicted failure to summit, but did not increase risk of developing AMS. AMS is a common cause of morbidity on Mt. Kilimanjaro, and although the risk can be mitigated by a slower ascent, there is an ongoing need for education of individual trekkers, tour companies, and local authorities.
\end{abstract}

Key words: altitude sickness, mountain sickness, epidemiology

\section{Introduction}

Altitude illness commonly affects unacclimatized individuals traveling to altitudes over $2500 \mathrm{~m}$. The term refers to a number of diagnoses including acute mountain sickness (AMS), which is most common, as well as the more severe high altitude cerebral edema (HACE) and high altitude pulmonary edema (HAPE). ${ }^{1}$ AMS symptoms are nonspecific, with the diagnosis being defined as the presence of headache plus 1 or more of the following: gastrointestinal upset (anorexia, nausea, or vomiting), sleep disturbance, dizziness, and fatigue in an

Results of this study were presented by Dr James Lawrence at the Australasian College of Sports Physicians Annual Scientific Meeting, Coffs Harbour, NSW, Australia, February 2015.

Corresponding author: James S. Lawrence, BMedSci, BMBS, North Sydney Sports Medicine Centre, 60 Pacific Highway, St Leonards, NSW 2010, Australia (e-mail: drjameslawrence@gmail.com). individual recently arrived at altitude. ${ }^{2}$ The risk factors consistently associated with the development of all forms of altitude illness are rate of ascent and absolute altitude reached. ${ }^{3,4}$ Other risk factors for AMS that have been variably identified include younger age, female sex, smoking, and obesity., ${ }^{5,6}$ A previous history of AMS was not found to significantly correlate with increased susceptibility in a recent meta-analysis. ${ }^{7}$ Acetazolamide use has been found to be protective against AMS and HAPE and is regarded as a common option for prophylaxis. $^{8-10}$ When AMS symptoms are severe, descent is often required; if left untreated, AMS can progress to the life-threatening condition of HACE. ${ }^{11,12}$ HAPE can occur in combination with AMS/HACE, or in isolation. $^{13,14}$ Both HACE and HAPE require urgent descent and treatment.

Increasing numbers of tourists are travelling to high altitude environments globally, thus being exposed to 


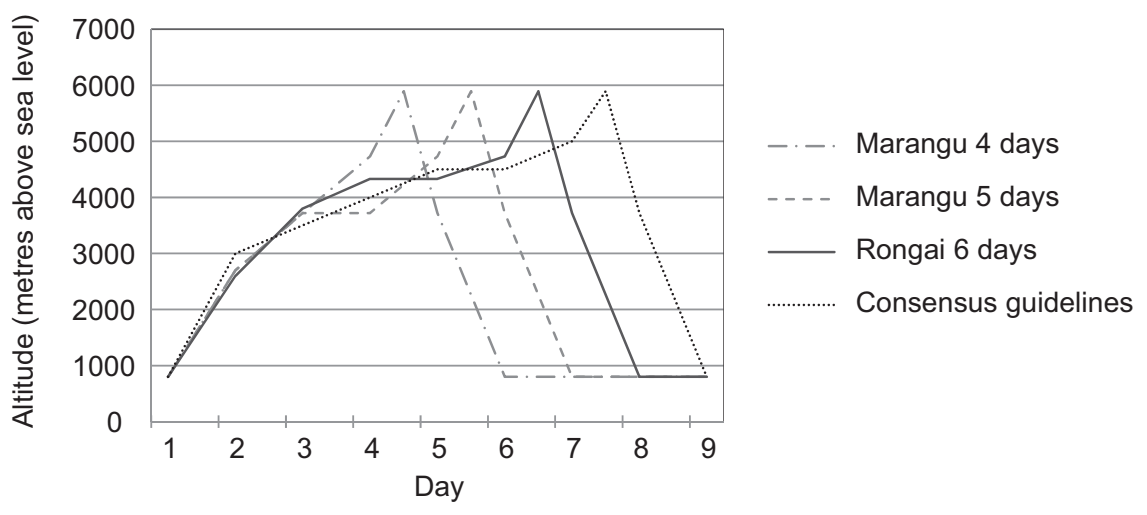

Figure 1. Diagrammatic representation of Mt. Kilimanjaro ascent profiles. The dotted line shows the minimum recommended ascent profile based on Wilderness Medical Society consensus guidelines. ${ }^{23}$

potentially significant risks. Mount Kilimanjaro, at 5895 $\mathrm{m}$, is the highest mountain in Africa and one of the "Seven Summits" (the highest mountains on each continent). It is easily accessible and the ascent is nontechnical, making it a popular tourist trekking destination. As a result, up to 40,000 climbers visit the mountain each year, with the majority of those reported to attempt the summit. ${ }^{15}$ There are multiple routes up Mount Kilimanjaro, which vary in their speed of ascent. The most popular routes take between 4 and 7 days to ascend, although longer treks are possible. Descent is typically over 2 days. The Marangu ("Coca-Cola") route has historically been the most popular ascent because it provides hut accommodation, rather than camping, and allows for the quickest climb, thus minimizing costs. On this route the summit is typically attempted on the fourth or fifth day. It is important to be aware of the variable terminology used when ascent profiles are described throughout the literature. For example, a 6-day route would be equivalent to a 4- or 5-day ascent, followed by a 1- or 2-day descent. ${ }^{16}$ The day on which the summit is attempted therefore most clearly defines the speed of the climb and will be used throughout this study.

The popularity and nontechnical nature of the Kilimanjaro trek belies the risks involved and the mountain tends to be underestimated. ${ }^{17,18}$ Previous studies describe very high rates of AMS on faster ascent profiles. Davies et $\mathrm{al}^{19}$ assessed trekkers on 4- and 5-day ascents, finding an overall AMS incidence of $77 \%$ (using Lake Louise score [LLS] $\geq 4$ ) and summit success of $61 \%$. There were no significant differences between 4- and 5day ascents. Davies et $\mathrm{al}^{19}$ lost 100 of 281 participants to follow-up. Therefore, their results for AMS incidence and summit failure may in reality have been underestimated. Karinen et $\mathrm{al}^{20}$ report a $75 \%$ incidence of AMS (LLS $\geq 3$ ) in 112 trekkers during a 5-day ascent on the Marangu route. Only $51 \%$ of this cohort reached the summit, defined as Gilman's Point (the crater rim at $5681 \mathrm{~m})$.

These climbing profiles have been criticized for their speed of ascent and associated high morbidity. ${ }^{21}$ Between 1996 and 2003, 25 tourist deaths were reported on Mt. Kilimanjaro, with 14 resulting from HAPE, HACE, or a combination. ${ }^{22}$ The Wilderness Medical Society consensus guidelines on AMS prevention recommend taking $\geq 2$ days to reach 3000 $\mathrm{m}$, followed by no more than a 500-m increase in sleeping altitude per day, with a rest day every 3 or 4 days. ${ }^{23}$ The ascent profiles of different routes on Mt. Kilimanjaro are shown in Figure 1. Alternative routes with slower ascent profiles, taking 6 or 7 days to reach the summit, have been suggested as safer alternatives, although there are currently very few data to support this. ${ }^{24}$ Nicol and Evans ${ }^{25}$ recently found a daily prevalence of AMS of up to $40 \%$ in a cohort of 25 trekkers on a 6-day ascent via the Lemosho Glades route, although they do not quote an overall incidence of AMS for the trek. Mackie and Windsor ${ }^{24}$ accompanied 12 trekkers on a 7-day ascent on the same route and report $91 \%$ reaching the summit with only 2 climbers experiencing AMS.

To date there have been no studies investigating the incidence of AMS in trekkers climbing the Rongai Route up Mt. Kilimanjaro, with the summit being attempted on day 6. The purpose of this study, therefore, was to investigate the occurrence of altitude illness and summit success among trekkers on this increasingly popular ascent profile. We hypothesized that the results would compare favorably with those for more rapid climbing profiles.

\section{Methods}

We conducted a prospective cohort study to assess the incidence of AMS and rate of summit success on a 6-day 
ascent of Mt. Kilimanjaro. Subjects consisted of all trekkers undertaking the climb through a single Australian adventure travel company between August 2012 and July 2014. All participants were provided with written information regarding the study, altitude sickness, and preparation for the trek in the months before their departure. Information on acetazolamide use was provided, including potential side effects, contraindications to use, and dosage regimens. Individuals contemplating its use prophylactically were advised to discuss the option with their general practitioner. All trekkers were given medical clearance to undertake the expedition by their general practitioner, as per the company's medical policy. There was potential for a climber to be deemed medically unfit to undertake the trip; however, no participant was excluded based on perceived level of fitness alone. Each group was fundraising for charity and ranged in size from 10 to 25 people. A tour leader and an expedition doctor accompanied each group and were excluded from the study, because their ability to summit was dependent on other individuals.

All trekkers were lowland dwellers in Australia and arrived in Tanzania with no prior opportunity for acclimatization. Groups ascended Mt. Kilimanjaro via the Rongai route, leaving Moshi $(\sim 800 \mathrm{~m})$ on day 1 , with camps at $2600 \mathrm{~m}$ (1 night), 3800m (1 night), 4330 $\mathrm{m}$ (2 nights, permitting an additional day for acclimatization; see Figure 1), and $4730 \mathrm{~m}$ (1 night). The summit $(5895 \mathrm{~m})$ was attempted early in the morning of day 6 . After the summit attempt, climbers returned to base camp at $4730 \mathrm{~m}$ for a meal before descending to $3800 \mathrm{~m}$ for the last camp. The total length of the trek was 8 days.

Baseline data were collected on all participants including age, sex, height, weight, and use of prophylactic acetazolamide (dose and start date). Trekkers were evaluated using the LLS, a questionnaire that rates the severity of 5 symptoms of AMS: headache, gastrointestinal symptoms, fatigue/weakness, dizziness/lightheadedness, and difficulty sleeping. ${ }^{26}$ Each item is rated from 0 to 3 , giving a maximum score of 15 . In its original description, a diagnosis of AMS was defined as the presence of headache, plus at least 1 other symptom, and a total score of $\geq 3$. More recently, it has been suggested that a LLS of $\geq 5$ more closely correlates with other AMS scoring systems, and thus both thresholds were used in our data analysis. ${ }^{6}$ The expedition doctor recorded the total LLS for individual trekkers each morning before leaving camp and documented whether that individual met the criteria for a diagnosis of AMS (ie, presence of headache, at least 1 other symptom, and a total score $\geq 3$ ). Scores for each subset of the questionnaire were not recorded to simplify the documentation process while on the mountainside. No analysis was therefore conducted on individual symptoms within the LLS questionnaire, something that future studies should consider including. On summit night it is logistically very difficult to record scores during the summit attempt. The group can often fragment and there is a need to minimize time exposed to both extreme altitude $(>5500 \mathrm{~m})$ and potential adverse weather while at the summit. LLS for day 6 were therefore recorded at base camp $(4730 \mathrm{~m})$, after return from the summit attempt.

Statistical analyses were performed using SPSS version 21 (IBM, Armonk, NY). Frequencies and 95\% confidence intervals (CI) were computed. Univariate associations of predictors of AMS (LLS $\geq 3$ and LLS $\geq 5$ ) and failure to summit were investigated using contingency tables with a continuity corrected $\chi^{2}$ test. Logistic regression was used to investigate multivariate predictors using forward sequential analyses. Variables were retained in the model if $P<.05$ or if the odds ratio (OR) was $>2$.

Ethical approval was gained through the Australian Institute of Sport Ethics Committee (Approval No. 20131001), and written informed consent was gained from participants.

\section{Results}

Between August 2012 and July 2014 there were 13 groups that attempted the Mt. Kilimanjaro Rongai route trek, totaling 175 individual climbers, excluding the accompanying tour leaders and doctors. Of the 175 participants, 79 were women $(45.1 \%)$ and 96 were men $(54.9 \%)$. The age range was 19 to 69 years with a mean $( \pm \mathrm{SD})$ of $38 \pm 12$ years, and the body mass index (BMI) range was 18.3 to 35.1 with a mean $( \pm$ SD) of $25.0 \pm 3.4 \mathrm{~kg} / \mathrm{m}^{2}$. Acetazolamide use in our cohort was high, with $88 \%(154 / 175)$ of trekkers using it during the trek. Of those that took the medication, 96\% (148/154) used a dose of $125 \mathrm{mg}$ twice daily, whereas $4 \%(6 / 154)$ used a dose of $250 \mathrm{mg}$ twice daily. A number of medical co-morbidities were recorded, including asthma (10.3\%), hypertension $(5.1 \%)$, and depression or anxiety $(6.9 \%)$. No participants were taking regular nifedipine or oral corticosteroids. Trekkers were free to self-medicate with simple analgesics and nonsteroidal anti-inflammatories as required for musculoskeletal pain, headache, and so forth. Although this may have had a potential impact on AMS symptoms, it was considered to be consistent with normal practice among the general trekking population.

\section{AMS}

The incidence of AMS over the course of the trek using LLS $\geq 3$ was $52.6 \%(92 / 175)$. Using a cutoff of LLS 

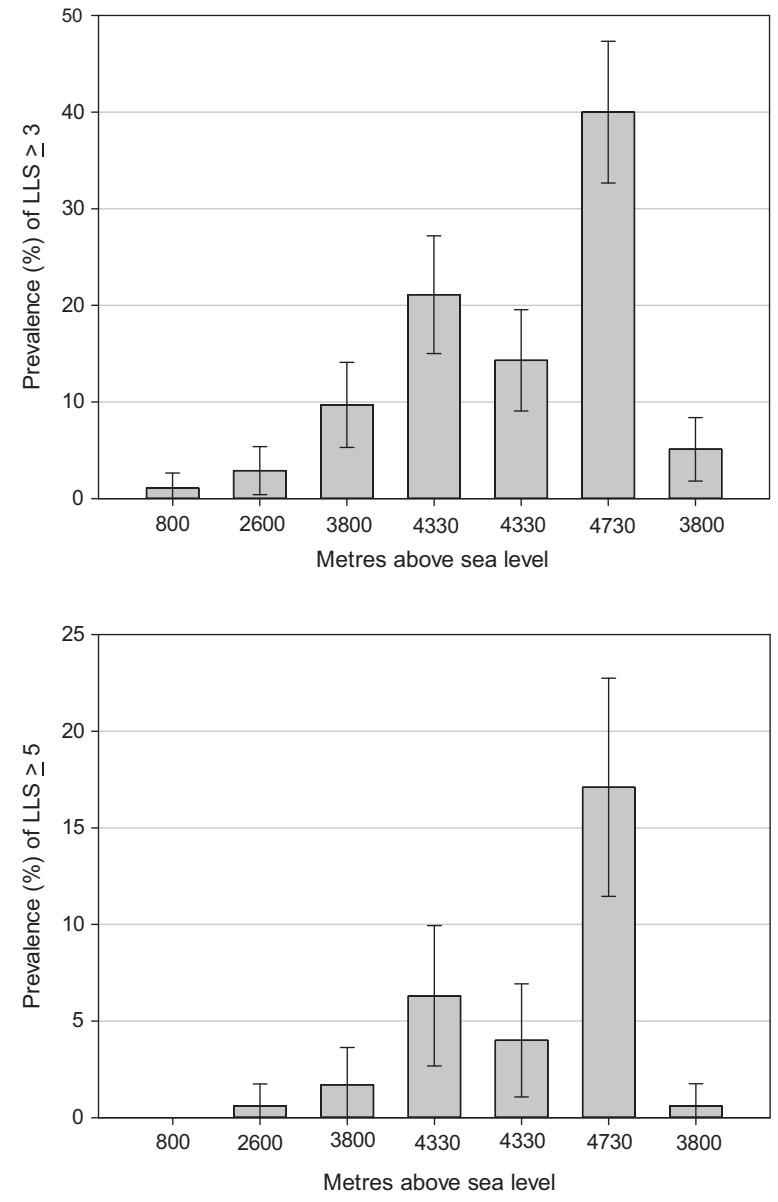

Figure 2. Daily prevalence of AMS (LLS $\geq 3$ and LLS $\geq 5$ ) at each camp throughout the trek. AMS, acute mountain sickness; LLS, Lake Louise score.

$\geq 5$, the incidence was $22.9 \%$ (40/175). The highest prevalence of AMS was on day 6 , after returning to base camp from the summit attempt, with a rate of $40.9 \%$ (70/ 171 ) and $17.5 \%$ (30/171) using LLS $\geq 3$ and $\geq 5$, respectively. Prevalence of AMS on a daily basis is shown in Figure 2. This demonstrates an increased rate of AMS with increasing altitude. There is a drop in prevalence after the acclimatization day at $4330 \mathrm{~m}$.
Using both LLS $\geq 3$ and LLS $\geq 5$, none of the risk factors we assessed (age, BMI, sex, or acetazolamide use) were found to significantly predict AMS (Table 1). No significant difference was demonstrated between dose of acetazolamide and LLS $\geq 3(P=.88)$ or LLS $\geq 5(P=.27)$.

Within our cohort, 1 participant $(0.6 \%)$ was diagnosed clinically with HAPE at an altitude of $2900 \mathrm{~m}$ on day 2. This person had marked dyspnea and reduced exercise capacity with tachycardia, tachypnea, and bilateral basal crepitus on auscultation. The trekker was treated with oral dexamethasone and immediate descent, after which there was complete resolution of symptoms, and no further treatment was required. Two other participants also required accompanied descent, both on day $3(\sim 3800 \mathrm{~m})$, with symptoms of severe AMS and early clinical signs of HACE (LLS of 6 and 7 on the morning of day 3 ). These 3 participants did not re-ascend.

\section{SUMMIT SUCCESS}

The overall summit success rate of our cohort was $88 \%$ $(154 / 175)$. Those reaching Gilman's Point $(5681 \mathrm{~m})$ or above, considered as a successful summit by some authors, totaled 165/175 (94\%). Of those that did not reach Gilman's Point, 7 trekkers turned back between base camp $(4700 \mathrm{~m})$ and Gilman's Point during the summit attempt. Three trekkers descended at earlier stages of the climb as detailed above.

Significant predictors of an unsuccessful summit attempt were age $\geq 40$ years (OR $7.81 ; 95 \%$ CI $2.61-$ 23.37; $P=.0002)$ and female sex (OR $8.55 ; 95 \%$ CI 2.63-27.84; $P=.0004$ ) (Table 2). Having a LLS $\geq 5$ at any stage during the trek (OR 7.02; 95\% CI 2.08-23.68; $P=.002)$ also significantly predicted failure to reach the summit, whereas when using a LLS $\geq 3$ significance was not achieved (OR 2.21; 95\% CI 0.73-6.67; $P=.161$ ). No significant difference was found between dose of acetazolamide and summit success $(P=.63)$.

Table 1. Logistic regression analysis of predictors for acute mountain sickness defined as LLS $\geq 3$ and LLS $\geq 5$

\begin{tabular}{|c|c|c|c|c|c|c|c|c|}
\hline \multirow[b]{3}{*}{ Variable } & \multicolumn{4}{|c|}{$L L S \geq 3$} & \multicolumn{4}{|c|}{$L L S \geq 5$} \\
\hline & \multicolumn{4}{|c|}{$95 \% C I$} & \multicolumn{4}{|c|}{$95 \% C I$} \\
\hline & $O R$ & Lower & Upper & $P$ value & $O R$ & Lower & Upper & $P$ value \\
\hline Female sex & 1.67 & 0.90 & 3.08 & .103 & 1.15 & 0.56 & 2.39 & .703 \\
\hline Age $>40$ years & 1.03 & 0.54 & 1.95 & .928 & 0.61 & 0.27 & 1.37 & .231 \\
\hline $\mathrm{BMI} \geq 30$ & 1.16 & 0.43 & 3.14 & .767 & 1.49 & 0.49 & 4.57 & .485 \\
\hline Acetazolamide use & 1.21 & 0.47 & 3.08 & .692 & 0.81 & 0.27 & 2.44 & .810 \\
\hline
\end{tabular}

LLS, Lake Louise score; CI, confidence interval; OR, odds ratio; BMI, body mass index. 
Table 2. Logistic regression of predictors for failure to reach the summit $(5895 \mathrm{~m})$

\begin{tabular}{lrrrr}
\hline & \multicolumn{5}{c}{ Failed summit attempt } \\
\cline { 2 - 5 } Variable & \multicolumn{4}{c}{$95 \%$ CI } \\
\cline { 2 - 5 } & OR & Lower & Upper & P value \\
\hline Female sex & 8.55 & 2.63 & 27.84 & .0004 \\
Age $>40$ years & 7.81 & 2.61 & 23.37 & .0002 \\
BMI $\geq 30$ & 2.17 & 0.52 & 9.03 & .287 \\
Acetazolamide use & 0.81 & 0.18 & 3.57 & .784 \\
Diagnosis of AMS (LLS $\geq 3)$ & 2.21 & 0.73 & 6.67 & .161 \\
Diagnosis of AMS (LLS $\geq 5)$ & 7.02 & 2.08 & 23.68 & .002 \\
\hline
\end{tabular}

CI, confidence interval; BMI, body mass index; AMS, acute mountain sickness; LLS, Lake Louise score.

\section{Discussion}

AMS

This study documents the largest cohort of climbers attempting to reach the summit of Mt. Kilimanjaro over 6 days. The first important finding is that on a 6-day ascent profile the incidence of AMS is still high (LLS $\geq 3,52.6 \%$; LLS $\geq 5,22.9 \%$ ). This reinforces the potential morbidity for tourists attempting to climb Mount Kilimanjaro. These figures are lower than those reported in previous studies looking at 4- and 5-day ascent profiles (75\% and $77 \%$ ) and are more in keeping with results from other mountain ranges. ${ }^{19,20}$ For example, recent findings from a similar altitude $(5400 \mathrm{~m})$ in the Himalayas reported that AMS incidence was $48 \%$ and $23 \%$ using LLS $\geq 3$ and LLS $\geq 5$, respectively. ${ }^{6}$

It is important to note that there is significant heterogeneity between the populations researched in the different published studies on Mt. Kilimanjaro, and therefore it is not valid to directly compare results. For instance, the use of acetazolamide varies widely. In the studies mentioned above, Davies et al ${ }^{19}$ reported $30 \%$ use, whereas Karinen et $\mathrm{al}^{20}$ documented only $9 \%$ use. Our high reported use of acetazolamide (88\%) is therefore a confounding factor in any direct comparison. The characteristics of individuals within previously studied groups, including fitness, age, BMI, and medical comorbidities are also not matched, and any comparison of summit success and AMS rates do not control for these multiple variables. Nevertheless, it remains valid to report that the results observed in our population favor a more successful outcome and lower incidence of AMS than previous studies on faster ascent profiles.

Daily prevalence rates for AMS on our trek increased with altitude as would be expected. However, it is important to note the drop in prevalence after the second night spent at $4330 \mathrm{~m}$. After the acclimatization day, prevalence of AMS reduced by $32 \%$ (LLS $\geq 3$ ) and $37 \%$ (LLS $\geq 5$ ). Although it is difficult to conclude that this drop is as a direct result of the acclimatization day, it does suggest that incorporating an extra night at the same altitude promotes some chance of symptomatic recovery from AMS. On this day participants typically undertake an acclimatization trek to a higher altitude before descending to the same camp for the night.

In our cohort, none of the assessed risk factors predicted development of AMS. This may be due to our relatively small sample size. However, these variables have not consistently been found to increase risk in other studies, and it therefore may reflect the ongoing uncertainty regarding their significance.

Our cohort had a high incidence of prophylactic acetazolamide use (88\%). Thus, the comparison group of "no acetazolamide use" had limited numbers, making it difficult to draw firm conclusions regarding acetazolamide effect. Statistically there was no difference in AMS or summit success rates between those who did and did not use acetazolamide. There was also no significant difference demonstrated between a dose of $125 \mathrm{mg}$ twice daily and $250 \mathrm{mg}$ twice daily. All participants were well informed about altitude sickness and the potential risks, as well as the potential use of acetazolamide as a prophylactic, which may account for the high rate of use. Anecdotally there was a high prevalence of the typical side effects of acetazolamide (diuresis, paraesthesia, distaste of carbonated drinks, and so forth), but no significant adverse effects were recorded from use of the medication. It has been suggested that acetazolamide use may reduce exercise capacity during the first 24 to 48 hours of altitude acclimatisation, particularly in those $>50$ years. $^{27}$ However, the implications of this on AMS incidence and ability of trekkers to summit on day 6 remain unclear.

Others have highlighted the difficulty of assessing AMS in the field, given the nonspecific nature of the symptoms, and we would echo this sentiment. ${ }^{28}$ Although LLS appears to be sensitive in detecting AMS, its specificity is likely to be low. It is, however, an easy tool to use and provides a quantifiable assessment of AMS, as well as ultimately reflecting the burden of symptoms at both an individual and group level. The experience from this study leads us to suggest that a threshold of LLS $\geq 5$, with presence of headache, is likely to be more specific to a true diagnosis of AMS than LLS $\geq 3$. It is also likely to offer more utility during an expedition, evidenced in this cohort by its association with failure to summit, as opposed to a LLS $\geq 3$. Practically it highlights those individuals during the trek who are likely to require closer monitoring, more 
support, and treatment for altitude-related illness. We note the conclusions of others that suggest the best system for AMS assessment remains undetermined and that further work to re-evaluate the optimal cut-offs for research is required. ${ }^{6,29,30}$

\section{SUMMIT SUCCESS}

The rate of summit success in our cohort was high, with $88 \%$ reaching Uhuru Peak (5895 $\mathrm{m}$ ), and $94 \%$ achieving Gilman's Point (5861 m) or above. This is in contrast to success rates of $51 \%$ and $61 \%$ in previous research on 4and 5-day ascents. ${ }^{19,20}$ A slower ascent profile is likely to be the biggest factor in this difference, but it is also important to note that our cohort consisted of groups of individuals who were well informed and well prepared for the trek. An accompanying doctor and tour leader offered support and reassurance en route, as well as providing symptomatic treatment and medical guidance as required. The fundraising nature of the trips may also have provided extra motivation and drive to achieve a successful climb. In addition, the use of acetazolamide was high among our cohort as previously discussed, although the impact of this on ability to summit is unclear.

Female sex and age $\geq 40$ were both independent risk factors for failure to summit, a new finding on Mt. Kilimanjaro. It should be noted that within these groups the summit success rate was still high, with $79.5 \%$ of women and $77.4 \%$ of those $\geq 40$ years reaching Uhuru Peak $(5895 \mathrm{~m})$. These groups did not have significantly higher rates of AMS, and therefore this does not account for their lower success rates. The possible explanations include both physical attributes of the individuals, such as general exercise capacity and endurance, as well as psychological factors such as risk taking behavior and willingness to continue with high fatigue levels and symptoms of AMS. Baseline data on exercise capacity was not collected.

Individuals diagnosed with AMS (LLS $\geq 5$ ) at any stage of the trek were less likely to summit, although this was not the case with LLS $\geq 3$. This would be expected, because if symptoms of AMS are persisting or worsening then further ascent should be halted and descent considered.

\section{LIMITATIONS}

The assessment of LLS was not conducted at the summit itself, but rather a few hours afterwards on return to base camp from the summit attempt. The true incidence of AMS may therefore have been slightly underestimated, although this is unlikely to have been by a significant amount. Only those trekkers who had recovered before returning to base camp would go unrecognized. Other studies to date have used a similar approach, because assessing LLS at the summit is impractical to do for a variety of reasons discussed previously.

Prophylactic acetazolamide use in our cohort was high. This limited the power of our statistical comparison, and we were therefore unable to find a difference in AMS incidence between those taking acetazolamide and those not. Despite this, however, it is feasible that the prophylaxis was effective and may have contributed to the lower incidence of AMS and higher summit success rate found in this study. It also limits any direct comparison of our outcomes to the results of prior studies with lower rates of acetazolamide use, as discussed previously.

\section{Conclusions}

We describe a lower incidence of AMS, and higher summit success rate, in a cohort of tourist trekkers on a 6-day ascent of Mt. Kilimanjaro than previously described on 4- and 5-day ascents. We speculate that an extra day allows for better acclimatization and promotes a safer and more successful trek, although a high use of acetazolamide in our group is a confounding variable. Despite a lower incidence, AMS remains common in our cohort and poses a significant risk of morbidity on Mt. Kilimanjaro. Age $\geq 40$ years and female sex are predictors for failure to summit; however, the chance of summiting remains high in these groups on a 6-day ascent. An LLS threshold of $\geq 5$ for the diagnosis of AMS may have greater practical utility, as in our cohort it was significantly associated with reduced summit success.

\section{Acknowledgments}

This study was conducted with the cooperation of Inspired Adventures Pty Ltd. The authors give special thanks to the expedition doctors that assisted in data collection for this study: Drs Rebecca Davies, Stephen Skinner, Pieter Peach, Ruth Attard, Phillip Hall, Justine Douthwaite, Claudia Lee, Danielle Wood, Caitlin Keighley, Kirsten Cameron, Naomi Abdullah, and Shaun Counsell.

\section{References}

1. Basnyat B, Murdoch DR. High-altitude illness. Lancet. 2003;361:1967-1974.

2. Hackett PH, Roach RC. High-altitude illness. N Engl J Med. 2001;345:107-114. 
3. Bloch KE, Turk AJ, Maggiorini M. Effect of ascent protocol on acute mountain sickness and success at Muztagh Ata, 7546 m. High Alt Med Biol. 2009;10:25-32.

4. Davis PR, Pattinson K, Mason NP. High altitude illness. $J$ R Army Med Corps. 2011;157:12-17.

5. Gaillard S, Dellasanta P, Loutan L, Kayser B. Awareness, prevalence, medication use, and risk factors of acute mountain sickness in tourists trekking around the Annapurnas in Nepal: a 12-year follow-up. High Alt Med Biol. 2004;5:410-419.

6. McDevitt M, McIntosh SE, Rodway G, Peelay J, Adams DL, Kayser B. Risk determinants of acute mountain sickness in trekkers in the Nepali Himalaya: a 24-year follow-up. Wilderness Environ Med. 2014;25:152-159.

7. MacInnis MJ, Lohse KR, Strong JK, Koehle MS. Is previous history a reliable predictor for acute mountain sickness susceptibility? A meta-analysis of diagnostic accuracy. Br J Sports Med. 2014;49:69-75.

8. Gertsch JH. Randomised, double blind, placebo controlled comparison of ginkgo biloba and acetazolamide for prevention of acute mountain sickness among Himalayan trekkers: the prevention of high altitude illness trial (PHAIT). BMJ. 2004;328:797.

9. Kayser B, Dumont L, Lysakowski C, Combescure C, Haller G, Tramèr MR. Reappraisal of acetazolamide for the prevention of acute mountain sickness: a systematic review and meta-analysis. High Alt Med Biol. 2012;13: 82-92.

10. Low EV, Avery AJ, Gupta V, Schedlbauer A, Grocott MPW. Identifying the lowest effective dose of acetazolamide for the prophylaxis of acute mountain sickness: systematic review and meta-analysis. BMJ. 2012;345:e6779.

11. Imray $\mathrm{C}$, Booth $\mathrm{A}$, Wright $\mathrm{A}$, Bradwell A. Acute altitude illnesses. BMJ. 2011;343:d4943.

12. Hackett PH, Roach RC. High altitude cerebral edema. High Alt Med Biol. 2004;5:136-146.

13. Stream JO, Grissom CK. Update on high-altitude pulmonary edema: pathogenesis, prevention, and treatment. Wilderness Environ Med. 2008;19:293-303.

14. Hall DP, Duncan K, Baillie JK. High altitude pulmonary oedema. J R Army Med Corps. 2011;157:68-72.

15. Christie I, Fernandes E, Messerli H, Twining-Ward L. Tourism in Africa: Harnessing Tourism for Growth and Improved Livelihoods. Washington, DC: The World Bank; 2013;229-231.

16. Meyer J. Twice-daily assessment of trekkers on Kilimanjaro's Machame route to evaluate the incidence and time-course of acute mountain sickness. High Alt Med Biol. 2012;13: 281-284.

17. Bellis F. Tour operators should tell clients about potential risks of adventure holidays. BMJ. 2002;324:1040.

18. Welch P, Symmons D. The dangers of trekking on Mount Kilimanjaro. J Travel Med. 2013;20:137.

19. Davies AJ, Kalson NS, Stokes S, et al. Determinants of summiting success and acute mountain sickness on Mt. Kilimanjaro (5895 m). Wilderness Environ Med. 2009;20:311-317.

20. Karinen H, Peltonen J, Tikkanen H. Prevalence of acute mountain sickness among Finnish trekkers on Mount Kilimanjaro, Tanzania: an observational study. High Alt Med Biol. 2008;9:301-306.

21. Toovey S, Jamieson A. Tourists travelling to Kilimanjaro do need advice. BMJ. 2002;325:46.

22. Hauser M, Mueller A, Swai B, Moshi E, Ole Nguyaine S. Deaths due to high altitude illness among tourists climbing Mt. Kilimanjaro. Proceedings of the 2004 South African Travel Medicine Society. February 9-11, 2004; Cape Town, South Africa.

23. Luks AM, McIntosh SE, Grissom CK, et al. Wilderness Medical Society Consensus Guidelines for the prevention and treatment of acute altitude illness. Wilderness Environ Med. 2010;21:146-155.

24. Mackie S, Windsor JS. Is there a safer and more successful way to climb Kilimanjaro? High Alt Med Biol. 2009;10: 301-303.

25. Nicol LG, Evans HE. Walk high, sleep low: an observational cohort study of altitude symptoms and physiological profiles over a 6 day ascent of Mount Kilimanjaro. Wilderness Environ Med. 2014;25:354.

26. Savourey G, Guinet A, Besnard Y, Garcia N, Hanniquet AM, Bittel J. Evaluation of the Lake Louise acute mountain sickness scoring system in a hypobaric chamber. Aviat Space Environ Med. 1995;66:963-967.

27. Bradwell AR, Myers SD, Beazley M, et al. Exercise limitation of acetazolamide at altitude $(3459 \mathrm{~m})$. Wilderness Environ Med. 2014;25:272-277.

28. Roach R, Kayser B. Measuring mountain maladies. High Alt Med Biol. 2007;8:171-172.

29. Wagner DR, Teramoto M, Knott JR, Fry JP. Comparison of scoring systems for assessment of acute mountain sickness. High Alt Med Biol. 2012;13:245-251.

30. Dellasanta P, Gaillard S, Loutan L, Kayser B. Comparing questionnaires for the assessment of acute mountain sickness. High Alt Med Biol. 2007;8:184-191. 